\title{
Reply to the correspondence letter by Sumeet Gulshan Dua: "Are great toes always abnormal in patients with fibrodysplasia ossificans progressiva?"
}

\author{
Mutlu Kartal-Kaess
}

Received: 18 January 2011 / Accepted: 19 January 2011 / Published online: 12 February 2011

(C) Springer-Verlag 2011

Sir,

We thank Summeet Gulshan Dua and Nilendu C. Purandare for their astute comments on our recent paper in which we reported a patient with classical fibrodysplasia ossificans progressiva (FOP) [1]. Our report insisted on the importance of inspecting the great toes in children with heterotopic ossifications because this anatomical detail helps to establish the diagnosis of FOP.

The two authors refer to some other recent studies describing FOP variants with normal great toes. In addition, they describe a patient of their own with heterotopic calcifications, an osteochondroma, and normal toes. They made the diagnosis of FOP variant according to the clinical and radiological features of their patient. They conclude that FOP cannot be ruled out in patients with normal great toes.

We would like to make some important remarks. First, the authors clearly state that their patient has a FOP variant. It is, however, not clear how old the patient is and at what age the first clinical manifestations occurred. Second, it is also not mentioned whether the patient carries a mutation in the ACVRI gene, and if so, whether it is the current c. $617 \mathrm{G}>\mathrm{A} ; \mathrm{p} . \mathrm{R} 2 \mathrm{O} 6 \mathrm{H}$ or a novel one.

We feel that the authors missed an important point: all patients with classical FOP (i.e., 98\% of all the patients with any form of FOP) have malformed toes. Yet, we are all aware of extremely rare variants, but most diagnostic errors have been in the pediatric age. If children have evanescent soft tissue swellings, the toes must be inspected. Patients with FOP-plus or FOP variants may have more severe toe malformations or none at all. However, we like to stress once again: all patients with classical FOP have malformed great toes.

\section{Reference}

1. Kartal M, Shore EM, Xu M et al (2010) Fibrodysplasia ossificans progressiva: watch the great toes! Eur J Pediatr 169:1417-1421 